\title{
New families of exact traveling wave solutions to the van der Waals p-system
}

\author{
Muhammad Bilal ${ }^{1}$, Muhammad younis ${ }^{2}$, Hadi Rezazadeh ${ }^{3}$, Tukur Sulaiman ${ }^{4}$, Shafqat \\ Rehman $^{1}$, and USMAN YOUNAS ${ }^{5}$ \\ ${ }^{1}$ University of the Punjab \\ ${ }^{2}$ PUCIT \\ ${ }^{3}$ Faculty of Engineering Technology, Amol University of Special Modern Technologies \\ ${ }^{4}$ Firat University Elazig, Turkey \\ ${ }^{5}$ Punjab University
}

October 12,2020

\begin{abstract}
In this article, we successfully construct various kinds of exact traveling wave solutions like, hyperbolic (kink and singular kink-shaped soliton), trigonometric (periodic and singular periodic) as well as rational function solutions for the best known mixed hyperbolic-elliptic system of conservation laws, namely the van der Waals gas system in the viscosity-capillarity regularization version by means of $\left(\$ \backslash\right.$ frac $\left.\left\{\mathrm{G}^{\wedge}\right\}\left\{\mathrm{G}^{\wedge} 2\right\} \$\right)$-expansion and advanced $\$ \backslash \operatorname{mbox}\{\mathrm{e}\}^{\wedge}\{-\backslash \mathrm{phi}(\backslash$ tau $)\} \$$-expansion functions methods. These techniques are very useful and exceptionally helpful in a contrast with other analytical schemes, which show the effectiveness and the simplicity to discuss the exact solutions. 3D and contour figures are sketched in order to understand the physical movement of the gained results under the selections ofunknown parameters.
\end{abstract}

\section{Hosted file}

Manuscript File.pdf available at https://authorea.com/users/366453/articles/486214-newfamilies-of-exact-traveling-wave-solutions-to-the-van-der-waals-p-system 
figures/u1a/u1a-eps-converted-to.pdf 
figures/u1b/u1b-eps-converted-to.pdf 
figures/u1c/u1c-eps-converted-to.pdf 
figures/u1d/u1d-eps-converted-to.pdf 
figures/u9a/u9a-eps-converted-to.pdf 
figures/u9b/u9b-eps-converted-to.pdf 
figures/u9c/u9c-eps-converted-to.pdf 
figures/u9d/u9d-eps-converted-to.pdf 
figures/u11a/u11a-eps-converted-to.pdf 
figures/u11b/u11b-eps-converted-to.pdf 
figures/u11c/u11c-eps-converted-to.pdf 
figures/u11d/u11d-eps-converted-to.pdf 
figures/u19a/u19a-eps-converted-to.pdf 
figures/u19b/u19b-eps-converted-to.pdf 
figures/u19c/u19c-eps-converted-to.pdf 
figures/u19d/u19d-eps-converted-to.pdf 